\title{
Utilidad del índice tobillo-brazo en el traumatismo de extremidad inferior con signos blandos
}

\section{Usefulness of ankle-brachial index in lower extremity trauma with soft signs}

\author{
Rodolfo Alcalá-de Loza*, Claudia E. Mojica-Rodríguez y Sergio Guzmán-Lepe \\ Servicio de Cirugía Vascular, Hospital Civil de Guadalajara, Guadalajara, Jalisco, México
}

\begin{abstract}
Resumen
Antecedentes: La violencia en el país de las últimas décadas ha incrementado el número de lesiones vasculares, la morbimortalidad de la población y los costos de los servicios de salud. Objetivos: Determinar la utilidad del índice tobillo-brazo en pacientes que sufren traumatismo de extremidad inferior con signos blandos. Métodos: Se realizó un estudio prospectivo, analítico y descriptivo de julio de 2018 a julio de 2020 en el Hospital Civil Fray Antonio Alcalde, en individuos que cumplieran criterios de inclusión. Los datos se obtuvieron de las historias clínicas y la exploración física. Se realizó un análisis estadístico con medidas de tendencia central y dispersión, frecuencias y porcentajes, con significancia estadística $p<0.05$. Resultados: 72 pacientes con 76 extremidades lesionadas, 65 con ITB inicial $>0.9,7<0.9$, estos últimos sometidos a intervención quirúrgica; los 65 sujetos $>0.9$ se siguieron a las 6 horas con nuevo ITB; 61 tuvieron $>0.9$ y se descartó una lesión y $4<0.9$ fueron objeto de intervención; se relacionó el ITB $<0.9$ con la afectación del área femoropoplítea $(p<0.001)$. Conclusiones: El índice tobillo-brazo es útil como detección de lesiones vasculares en la zona femoropoplitea, ante la ausencia de estudios de gabinete en la unidad hospitalaria.
\end{abstract}

Palabras clave: Índice tobillo-brazo. Lesión vascular. Signos blandos. Extremidad inferior.

\begin{abstract}
Background: Violence in the country in recent decades has generated an increase in vascular injuries, producing an impact on the morbidity and mortality of the population and costs in health services. Objectives: to determine the usefulness of the ankle-brachial index in patients with lower extremity trauma and soft signs. Methods: a prospective, analytical and descriptive study was carried out from July 2018-July 2020 at Hospital Civil Fray Antonio Alcalde, which met inclusion criteria. The data obtained from the medical history and physical examination. Performing statistical analysis with measures of central tendency and dispersion, frequencies and percentages, with statistical significance $p<0.05$. Results: 72 patients with 76 injured limbs, 65 with initial $A B I>0.9,7<0.9$; The last ones underwent surgery, the 65 subjects $>0.9$ were followed up at 6 hours with a new $A B I, 61$ presented $>0.9$ ruling out injury and $4<0.9$ which were intervened, the presence of $A B I<0.9$ was associated with the involvement of the femoropopliteal sector $(p<0.001)$. Conclusions: the ankle-brachial index is useful as a screening for vascular lesions in the femoropopliteal sector, in the absence of an office study in the hospital unit.
\end{abstract}

Key words: Ankle brachial index. Vascular injury. Soft signs. Lower limb.

Correspondencia:

${ }^{\star}$ Rodolfo Alcalá-de Loza

E-mail: dr.rodolfoalcala@gmail.com

DOI: 10.24875/RMA.20000036

0377-4740/@ 2020 Sociedad Mexicana de Angiología y Cirugía Vascular y Endovascular, A.C. bajo la licencia CC BY-NC-ND license (http://creativecommons.org/licenses/by-nc-nd/4.0/).
Available online: 27-01-2021 Rev Mex Angiol. 2020;48(4):117-122

Wublicado por Permanyer. Este es un artículo open access

\footnotetext{
(1)
} 


\section{Introducción}

El traumatismo es una herida o lesión que se caracteriza por una alteración estructural o desequilibrio fisiológico consecutivos a la exposición aguda a energía mecánica, térmica, eléctrica o química, o por la ausencia de elementos esenciales, como el calor u oxígeno'. Las lesiones vasculares pueden determinar la pérdida de una extremidad y pueden causar la muerte o contribuir a ella en quienes sufren traumatismos graves 0 múltiples ${ }^{2}$. Las consecuencias de una lesión vascular dependen del mecanismo ejercido para infligir el daño, el sitio de la lesión, las lesiones adjuntas, un correcto diagnóstico temprano y un tratamiento o reparación tan satisfactorios como las condiciones lo permitan ${ }^{3}$.

Los traumatismos vasculares constituyen una emergencia evidentemente quirúrgica que se ha convertido en un problema de salud pública en el mundo entero, en particular en México, por el aumento progresivo de casos en la práctica civil. Es una de las evidencias más claras de violencia urbana y militar que posee mayor gravedad y un reto para los cirujanos que deben afrontar con una actitud especial, fundamentada en bases sólidas de entrenamiento quirúrgico y conceptuales ${ }^{4}$.

El desarrollo de esta disciplina proviene de la enseñanza y experiencia de los cirujanos militares durante los grandes conflictos bélicos de este siglo: guerras mundiales, Guerra de Vietnam, Guerra de Corea, etc. El terrorismo, los grupos paramilitares y guerrillas, las mafias, el aumento de los accidentes de tránsito como una de las consecuencias del desarrollo tecnológico han provocado el aumento de la prevalencia de estas lesiones en la práctica civil hasta igualar su frecuencia durante la guerra ${ }^{5}$.

El tratamiento del traumatismo vascular se actualiza de forma continua, en especial en el área más común de las lesiones vasculares, las extremidades. Varios aspectos del diagnóstico y tratamiento del traumatismo vascular son tema de debate aún en la actualidad ${ }^{6}$. La incidencia y prevalencia del traumatismo vascular se vinculan con factores sociales y culturales: conflictos armados, grado de industrialización, índices de violencia; sin embargo, los cálculos son poco reales debido a la gravedad de los casos, toda vez que las víctimas mueren muchas ocasiones en el lugar del accidente por la localización, magnitud de sangrado y retraso de la atención. En un plano mundial, las lesiones vasculares en hospitales no militares representan $0.65 \%$ del total de ingresos al año en los servicios de urgencias, de los cuales $88 \%$ afecta al género masculino, con una proporción de 12:1 respecto del género femenino ${ }^{7}$. Un punto de análisis importante es el incremento de las lesiones vasculares yatrogénicas por el aumento de las intervenciones de cirugía de mínima invasión, procedimientos invasivos como la colocación de catéteres y lesiones durante el tratamiento quirúrgico abierto. Todas estas anomalías se atienden en grandes centros hospitalarios que incluyen programas de formación de recursos humanos en salud 8 .

El traumatismo vascular es una complicación mayor de los traumatismos militares y civiles. Los traumatismos vasculares periféricos (TVP) representan al menos 3\% de los daños traumáticos, pueden ser devastadores y conducir a la pérdida de una extremidad y aun la muerte. Esto es especialmente cierto cuando se combinan varias lesiones en la extremidad, con compromiso de piel, hueso, músculos y nervios y, por otro lado, es dependiente de un diagnóstico oportuno y un tratamiento adecuado. El traumatismo se ha convertido en un problema de salud pública en muchas partes del mundo y su variante vascular es un importante componente de este problema. El traumatismo vascular periférico afecta a personas jóvenes de 20 a 40 años debido sobre todo a la prevalencia de los traumatismos en este grupo de edad mundial". "El incremento de la violencia en México está generando un aumento de la incidencia de lesiones vasculares periféricas. México es un país con una alta prevalencia de violencia en la última década". Se ha convertido en un escenario en el cual abundan los traumatismos vasculares periféricos y la deficiencia de recursos diagnósticos y terapéuticos es un verdadero desafío para el tratamiento adecuado de las lesiones vasculares. La valoración clínica muchas veces es el único recurso diagnóstico disponible ${ }^{8}$.

"En México el traumatismo se ha convertido en un problema crítico de salud, se estima que las defunciones asociadas a traumatismo ocupan la cuarta posición como causa de mortalidad general"10 y la primera como causa de fallecimiento en personas económicamente activas. En el país, las lesiones vasculares constituyen uno de los problemas más grandes y en crecimiento desde el inicio de los conflictos armados por la disputa de territorios entre los diversos cárteles de la droga, pero es difícil por desgracia establecer adecuadamente su epidemiología, que puede ser apenas menor a la registrada en Estados Unidos (3\% de todos los casos de traumatismo mayor se relaciona con lesión vascular o éstos son sólo vasculares); se ha observado un incremento relacionado con la mayor inseguridad, inequidad económica y desarrollo, acceso "más fácil" a armas de alto poder, todo lo cual ha favorecido un aumento sostenido de la frecuencia de lesiones por proyectil de 
arma de fuego y objetos punzocortantes ${ }^{8}$. Es de suma importancia conocer la epidemiología de este grupo de pacientes en México, ya que las lesiones a vasos periféricos y centrales tienen un potencial alto de morbimortalidad si no se reconocen o tratan de forma oportuna; el tratamiento adecuado puede alcanzar tasas de salvamento de extremidades inferiores de $83.8 \%$ y de supervivencia de $98.5 \%{ }^{6}$.

No obstante, la lesión vascular puede no tener características clínicas evidentes y aparentes, como las ocurridas en las heridas por arma de fuego, en trayectos cercanos a vasos de las extremidades, según el fenómeno de cavitación explicado en balística. En realidad, la exploración física falsa negativa puede representar $10 \%$ a $35 \%$ de los casos, ya que es posible una lesión vascular no oclusiva ${ }^{11}$.

Ante un traumatismo de extremidad inferior es preciso reconocer los signos duros y blandos, con especial atención en estos últimos. "En estos casos, la posibilidad de que exista un traumatismo vascular significativo es menor al $5 \%$, por lo que en general no hay necesidad de realizar exámenes complementarios sino que pueden ser tamizados por medio del índice tobillo-brazo o dejar en observación y evaluar si aparecen signos duros." 4

Estas conclusiones hacen más controversial aún elegir el método de valoración apropiado para el traumatismo vascular periférico. Las lesiones vasculares periféricas clínicamente ocultas tienen una tasa de incidencia de $10 \%$ a $17 \%$ en gran parte de las series publicadas 4 .

Ante esta situación, este trabajo de investigación intenta valorar la utilidad del índice tobillo-brazo como método diagnóstico rápido al alcance del profesional de salud en relación con la cirugía vascular; el objetivo es descartar con facilidad lesiones vasculares ocultas ante la exploración física inicial en pacientes con traumatismo de extremidad inferior y signos blandos; esto favorecerá el diagnóstico oportuno y evitará complicaciones y discapacidades.

\section{Métodos}

Este trabajo es un estudio descriptivo, analítico y prospectivo que tiene como centro el Hospital Civil de Guadalajara Fray Antonio Alcalde en la ciudad de Guadalajara, México, y comprende un período de 24 meses, entre julio de 2018 y julio de 2020; se integró con pacientes ingresados con traumatismo de extremidad inferior y signos blandos, hasta conformar una población de estudio de 72 pacientes y 76 extremidades

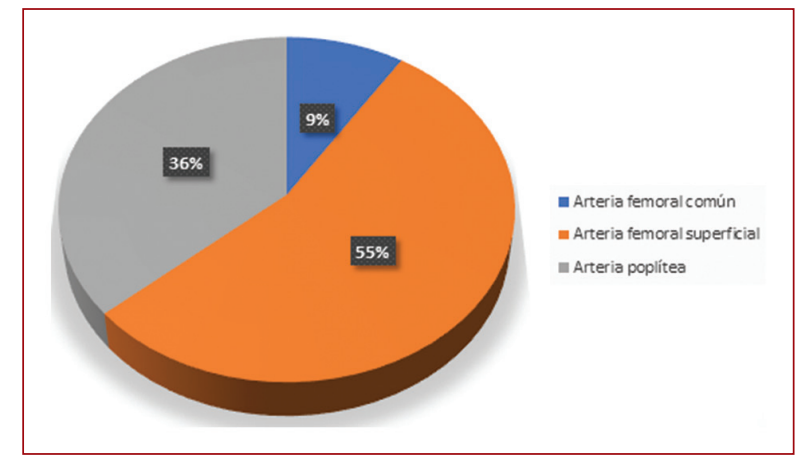

Figura 1. Distribución de la arteria afectada en la extremidad inferior.

inferiores lesionadas que cumplieron criterios de inclusión. Se realizó medición del índice tobillo-brazo en la valoración en el servicio de urgencias y se estableció seguimiento en los pacientes con valor $>0.9$, con nueva medición 6 horas después de la valoración inicial.

Las variables de estudio se obtuvieron de acuerdo con el formato de recolección de datos, provenientes del registro, historia clínica y exploración física de los pacientes, que se halla en la base de datos del departamento de archivo de esta unidad hospitalaria. Se recolectó la información con las variables relativas a edad, género, índice tobillo-brazo, extremidad lesionada, mecanismo de lesión, localización de la herida, signos blandos de lesión vascular, tipo de lesión, puntuación de MESS, horas de evolución, tiempo de espera para operación, tipo de intervención quirúrgica y complicaciones; se eliminó del estudio a los pacientes que no reunían los criterios de inclusión.

La información se obtuvo a través de instrumentos de recolección de datos, con análisis estadístico, pruebas estadísticas con medidas de tendencia central y dispersión, frecuencias y porcentajes; las variables cualitativas se analizaron mediante estadística no paramétrica, con un grado de significancia estadística $p<0.05$. Se representaron mediante gráficos y se utilizó el programa de evaluación estadística SPSS, versión 25.

\section{Resultados}

De los 72 pacientes del grupo de estudio se identificó una proporción de 9:1 en la relación hombre:mujer en la prevalencia de traumatismo de extremidad inferior; para la edad de pacientes afectados se encontró una moda de 21 años con media de 25 años (Fig. 1). En la evaluación de ITB inicial de los 72 sujetos de estudio se identificó lo siguiente: 7 pacientes $<0.9(9.7 \%)$ del 


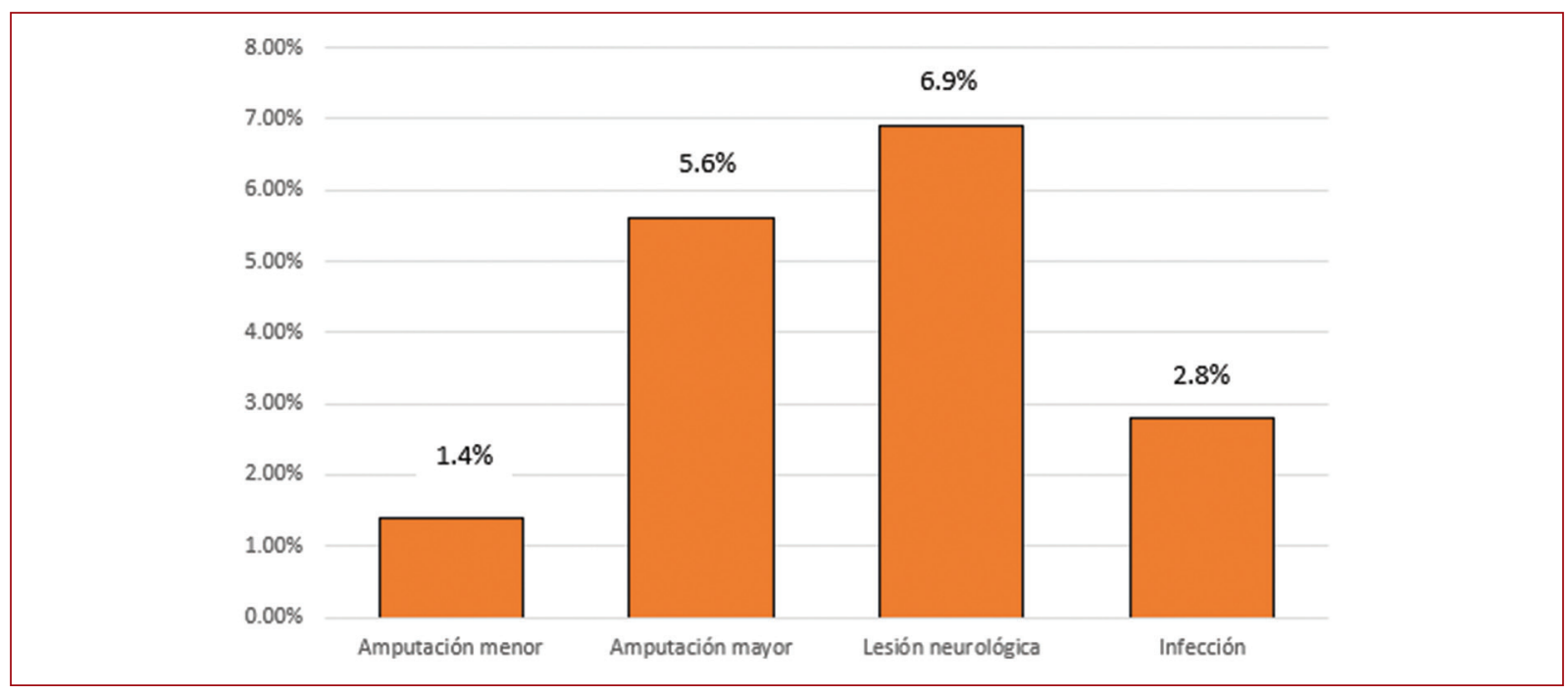

Figura 2. Complicaciones observadas en el grupo de estudio.

total se llevaron a intervención quirúrgica; 65 paciente con ITB > $0.9(90.3 \%)$ recibieron seguimiento a las 6 horas para nueva medición de ITB; a las 6 horas se identificó a 4 sujetos con ITB $<0.9(6.2 \%)$ que se llevaron a intervención; 61 individuos con ITB > 0.9 (93.8\%) se descartaron del estudio. Fue posible diagnosticar el $100 \%$ de las lesiones vasculares mediante este algoritmo, el $50 \%$ de los sujetos tenía dos o más signos blandos, la transección parcial o total fue la más frecuente $(27.3 \%)$, se encontró una puntuación de MESS de 4 puntos con mayor frecuencia (30.6\%), la media en el tiempo de evolución de la lesión fue de 6.86 horas, la media de tiempo de espera para intervención fue de 1.73 horas y el $63.6 \%$ fue objeto de derivación con injerto autólogo; los 11 pacientes con lesión vascular tuvieron complicaciones (Fig. 2). Asimismo, se observó que la lesión en la arteria poplítea requirió amputación con frecuencia mayor en el posoperatorio (Fig. 3); el valor de ITB $<0.9$ se vinculó con la aparición posterior de complicaciones $(p<0.05)$; se reconoció un nexo entre ITB $<0.9$ con la lesión vascular del sector arterial femoropoplíteo $(p<0.001)$ y la transección parcial o total se relacionó con más casos de amputación mayor $(p>0.43)$.

\section{Discusión}

De acuerdo con la prevalencia de traumatismos, la proporción 9:1 (hombre:mujer) corresponde a lo descrito en las publicaciones médicas; de igual modo, el grupo etario de la tercera a cuarta décadas de la vida identificado en los textos médicos corresponde a la media de edad de 25 años encontrada en este estudio; el tipo de lesión atendido con mayor frecuencia en más de la mitad de los casos es la lesión por proyectil de arma de fuego. Se demuestra también que el incremento de la violencia de esta región geográfica es consecuencia del rezago económico, social y cultural de América Latina; se trata de un grupo vulnerable a este tipo de lesiones, lo cual debería llevar a mejorar los protocolos de seguridad social para reducir la morbimortalidad de la población afectada.

En segundo lugar se encuentran las lesiones ocasionadas por accidentes de vehículo motor o motocicleta, que muestran una mayor exposición del grupo etario económicamente activo: es imprescindible mejorar y reforzar campañas de prevención en materia de vialidad.

En cuanto a los resultados encontrados acerca de la relación del valor ITB para diagnosticar lesión vascular se observó que las lesiones vasculares se presentaron ante un valor $<0.9$, lo que descarta a pacientes con valor $>0.9$ en el $100 \%$ de los casos mediante el algoritmo propuesto de valoración inicial y a las 6 horas de seguimiento. En cuanto a los signos blandos que muestra la extremidad lesionada se observó que la mitad de la población de estudio tuvo dos signos o más al momento de la valoración.

Se identificaron 11 casos de lesiones vasculares en pacientes con signos blandos en la población de estudio, todas diagnosticadas en la valoración inicial y a las 6 horas tras la revaloración. La mayoría de los pacientes al ingreso tuvo 4 puntos de MESS y la media de horas de evolución de la lesión para la correspondiente valoración fue de 6 horas, lo cual proporciona la pauta 


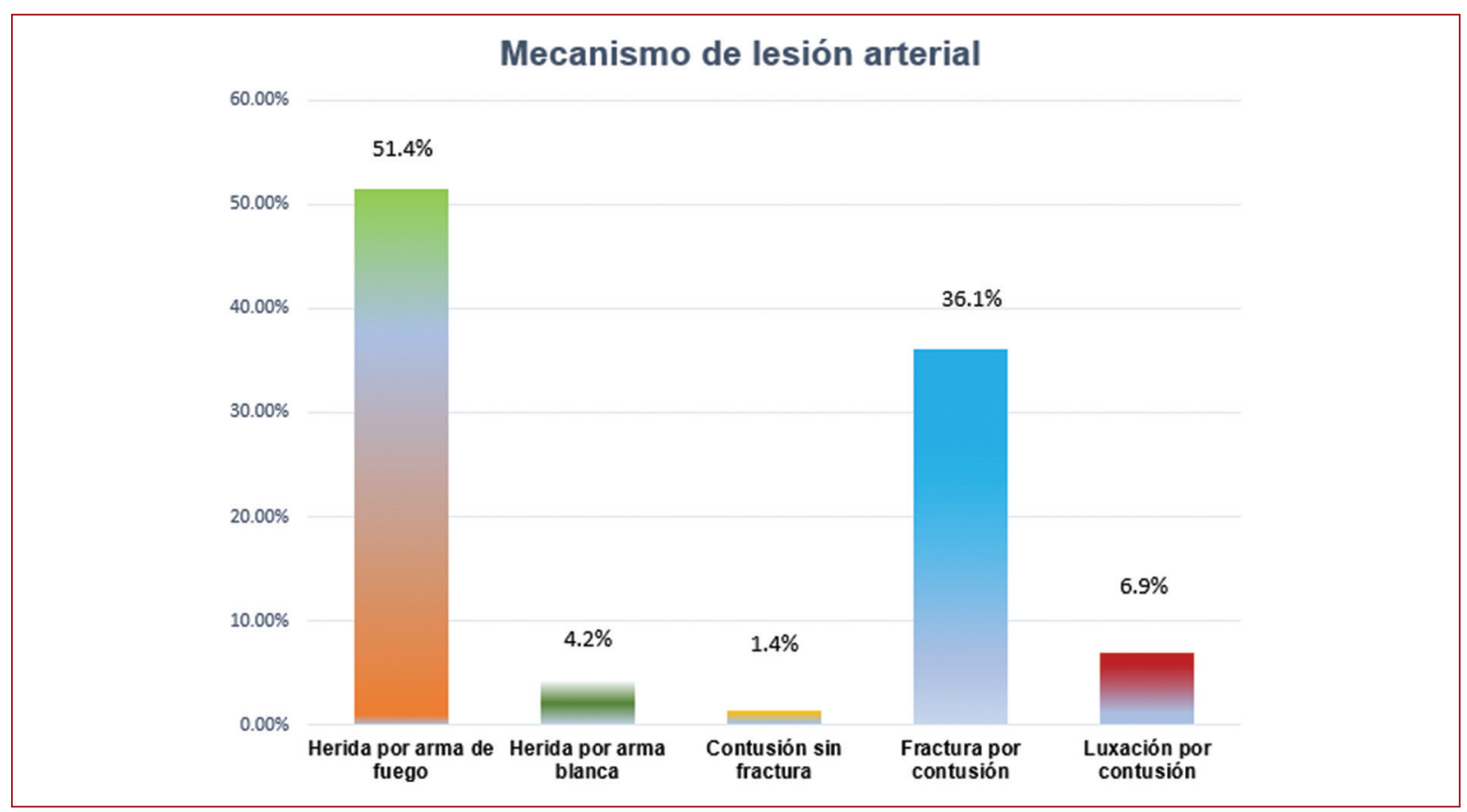

Figura 3. Mecanismo lesivo de las extremidades afectadas.

para mejorar y concientizar acerca del tiempo "de oro" para el salvamento de la extremidad inferior ante una lesión vascular; el objeto es disminuir la potencial morbilidad como lesión neurológica irreversible y la infección del sitio quirúrgico ante riesgo potencial de pérdida de extremidad, además de reducir también el costo económico para la unidad hospitalaria en cuanto a la estancia y la repercusión social que representa en la población económicamente activa. El tiempo de espera para la intervención quirúrgica en los pacientes diagnosticados con lesión vascular tuvo una media de 1 hora, lo cual demuestra que la valoración del triaje es adecuado. Se advirtió que el uso de injerto de vena safena se empleó con mayor frecuencia debido a lesiones arteriales poco complejas; todos los pacientes con lesión vascular tuvieron alguna complicación posquirúrgica, más a menudo lesión neurológica secundaria a factores como disección quirúrgica, tiempo de lesión y mecanismo de lesión. También se encontró que las lesiones vasculares de la arteria poplítea se relacionaron en este estudio con mayor prevalencia de amputación mayor. De igual manera, la arteria femoral superficial fue el sitio vascular más afectado, dado que la mayoría tuvo heridas penetrantes, lo cual concuerda con el hecho de que las heridas penetrantes de la extremidad inferior se vinculan muchas veces con lesiones en esa área, según las estadísticas de las publicaciones nacionales y mundiales.
Todos los pacientes con ITB $<0.9$ se correlacionaron con mayor riesgo de complicaciones posoperatorias; la zona femoropoplítea registró un ITB $<0.9$ al inicio y a las 6 horas de su medición; asimismo, la evolución de la lesión de 4 a 6 horas se acompañó de un mayor riesgo de complicaciones posquirúrgicas.

\section{Conclusiones}

El índice tobillo-brazo es útil para diagnosticar lesiones vasculares del área femoropoplítea y signos blandos con el algoritmo de una toma de ITB al inicio de la exploración y una a las 6 horas en pacientes que tuvieron un valor inicial $>0.9$, en las unidades hospitalarias que no cuentan con estudios de gabinete adecuados.

\section{Agradecimientos}

Los autores expresan un agradecimiento especial a cada uno de los profesores titulares por su apoyo moral, institucional y de seguimiento para la realizaciòn de este proyecto de investigación, que inició por la necesidad de atender a los pacientes de esta unidad hospitalaria que no cuentan con el apoyo económico para su abordaje y tratamiento. También se hallan en deuda con los compañeros y amigos del servicio de Cirugía Vascular y Endovascular del Hospital Civil Fray Antonio 
Alcalde por su apoyo en la recolección de datos para llevar a cabo este estudio de investigación.

\section{Conflicto de intereses}

No existe ningún conflicto de intereses.

\section{Financiamiento}

El financiamiento se obtuvo de manera propia con apoyo del servicio de angiología de la unidad hospitalaria.

\section{Responsabilidades éticas}

Protección de personas y animales. Los autores declaran que para esta investigación no se han realizado experimentos en seres humanos ni en animales.

Confidencialidad de los datos. Los autores declaran que han seguido los protocolos de su centro de trabajo sobre la publicación de datos de pacientes.
Derecho a la privacidad y consentimiento informado. Los autores han obtenido el consentimiento informado de los pacientes o sujetos referidos en el artículo. Este documento obra en poder del autor de correspondencia.

\section{Bibliografía}

1. Sidawy AN. Rutherford's vascular surgery and endovascular therapy. Philadelphia: Elsevier, 2018.

2. Salas C. Trauma vascular, visión del cirujano vascular. Rev Med Clin Condes. 2016;22(5): 686

3. Feliciano DV. Pitfalls in the management of peripheral vascular injuries Trauma Surgery \& Acute Open Acces. 2017:1-2.

4. Caicedo Valle J. Trauma vascular en extremidades: enfoque diagnóstico y terapéutico en urgencias. Medicina U.P.B. 2019; 38(1):57-66.

5. Gosselin RA, Spiegel A . Los traumas: el problema sanitario desatendido en los países en desarrollo. Boletín de la Organización Mundial de la Salud. 2009:246.

6. Rodríguez-López E. Trauma vascular civil: tres años de manejo en el Hospital General de México. Revista Mexicana de Angiología. 2017; 45(4):154-162.

7. Murilo R. Implications of vascular trauma in Brazil. 3rd ed. Trauma Rv. Philadelphia: Elsevier, 2016:333.

8. Moreno I, Borraez O, Ulloa J. Vascular trauma in Latinoamerica. Rich's vascular trauma. 3rd ed. Philadelphia: Elsevier; 2016:329.

9. Castañeda-Gaxiola R. El problema del trauma vascular en México. Cirugía General. 2000;22:254.

10. Instituto Nacional de Estadística, Geografía e Informática. Estadísticas Sociodemográficas. Morbimortalidad en México. INEGI. 2017:3.

11. Chong VE, Wayne SL. Applying peripheral vascular injury guidelines to penetrating trauma. J Surg Res. 2014;190(1):300-304. 